\title{
Urologists and clinical trials
}

\author{
Cora N Sternberg
}

There is no doubt that patients with genitourinary malignancies receive the best care when they are assessed and treated by a multidisciplinary, collaborating team of specialists; however, with regards to putting patients with urologic malignancies into clinical trials, this type of cooperation is often substantially lacking.

Urologists have already contributed thousands of patients with prostate cancer to screening and randomized treatment trials, which have resulted in new, clearly established guidelines on the roles of combined radiation treatment and hormonal therapy in patients with locally advanced disease, and of adjuvant radiation therapy after prostatectomy.

Trials in superficial bladder cancer have established important standards for intravesical therapy, but there is still a lot to be learned about genetic profiling, prognostic factors and who would benefit from immediate cystectomy. For muscle-invasive bladder cancer, neoadjuvant chemotherapy trials have taken years to accrue patients, and their results have been largely ignored by the medical community. Adjuvant bladder cancer trials have severely lacked in the collaboration of urologists and oncologists, and trials have really struggled to enroll patients.

Our perception of metastatic renal cell carcinoma has changed with the increasing knowledge of its biology and the advent of new drugs that have been studied in well-designed, randomized clinical trials. We still, however, urgently need to enroll patients into trials in order to understand the role of nephrectomy, combination therapy and sequencing in this new era.

So, why has it been so difficult for urologists and oncologists to collaborate and to conduct clinical trials similar to those that have been done in other cancer types, such as breast cancer? Surgeons and oncologists have defined the role of breast conservation and limited sentinel lymph node biopsy, and trials
As physicians, our united

front should

be collective

advocation

for all that

is needed

to promote

optimum care

for patients

with cancer.

CN Sternberg is Chair of the Department of Medical Oncology at the San Camillo and Forlanini Hospitals, Rome, Italy.

\section{Competing interests}

The author declared no competing interests.

www.nature.com/clinicalpractice doi:10.1038/ncpuro1125 that aim to reduce the dose of radiation are now underway. Gene profiling in breast cancer has been established, validated and used to drive clinical trials within large collaborative networks in Europe and in the US. Breast cancer physicians in Europe have united into a worldwide coalition of research groups, called TRANSBIG, to study 6,000 women in the MINDACT (Microarray In Node-negative Disease may Avoid ChemoTherapy) trial. With thousands of women enrolled or enrolling in clinical trials for breast cancer, is the issue that men are less willing to enter into trials than women? Are breast cancer specialists better organized than urologists? Or is more funding, public awareness and attention given to breast cancer research?

For years, urologists have felt self sufficient in dealing with many aspects of genitourinary oncology, which might partly be because few medical oncologists have devoted themselves to urology. With the increasing complexities of new therapies, there is a limit to how specialized a doctor can be. Interventional radiologists and medical and radiation oncologists have indeed become increasingly involved in this field, and some urologists could even feel threatened.

So, how can urologists ensure a commitment to the clinical trial? These goals can be accomplished through multidisciplinary collaboration, education, interaction at the fellowship training level and joint tumor boards that include urologists, oncologists, radiologists, pathologists and basic scientists. As physicians, our united front should be collective advocation for all that is needed to promote optimum care for patients with cancer. Integration of the urooncology team with equal partnership and no subordination, where each partner does what he knows best, constantly updating his knowledge of surgery and pathophysiology and imposing strict standards of quality control, is fundamental to achieving these goals. 